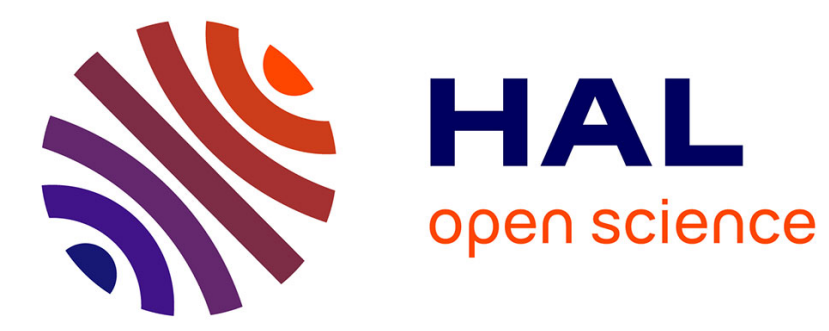

\title{
Suitability of Laser Doppler Velocimetry for the Calibration of Pressure Microphones
}

\author{
Anne Degroot, Robert Macdonald, Olivier Richoux, Bruno Gazengel, Murray
}

Campbell

\section{- To cite this version:}

Anne Degroot, Robert Macdonald, Olivier Richoux, Bruno Gazengel, Murray Campbell. Suitability of Laser Doppler Velocimetry for the Calibration of Pressure Microphones. Applied Acoustics, 2007, 69 (12), pp.1308-1317. 10.1016/j.apacoust.2007.09.003 . hal-00589266

\section{HAL Id: hal-00589266 https://hal.science/hal-00589266}

Submitted on 11 Jul 2011

HAL is a multi-disciplinary open access archive for the deposit and dissemination of scientific research documents, whether they are published or not. The documents may come from teaching and research institutions in France or abroad, or from public or private research centers.
L'archive ouverte pluridisciplinaire HAL, est destinée au dépôt et à la diffusion de documents scientifiques de niveau recherche, publiés ou non, émanant des établissements d'enseignement et de recherche français ou étrangers, des laboratoires publics ou privés. 


\title{
Suitability of Laser Doppler Velocimetry for the Calibration of Pressure Microphones
}

\author{
A. Degroot ${ }^{a}$, R. MacDonald ${ }^{b}$, O. Richoux ${ }^{a, *}$, B. Gazengel ${ }^{a}$, \\ M. Campbell ${ }^{\mathrm{b}}$ \\ ${ }^{a}$ Laboratoire d'Acoustique de l'Universit du Maine -UMR CNRS 6613-, \\ Av. O. Messiaen 72085 Le Mans, cedex 9 \\ ${ }^{\mathrm{b}}$ Acoustics and Fluid dynamics group, School of Physics, \\ University of Edinburgh Kings Buildings, Edinburgh
}

\begin{abstract}
The details of a new approach for absolute calibration of microphones, based on the direct measurement of acoustic particle velocity using Laser Doppler Velocimetry (LDV), are presented and discussed. The calibration technique is carried out inside a tube in which plane waves propagate and closed by a rigid termination. The method developed proposes to estimate the acoustic pressure with two velocity measurements and a physical model. Minimum theoretical uncertainties on the estimated pressure and minimum measurable pressure are calculated from the Cramer Rao Bounds on the estimated acoustic velocity amplitude and phase. These uncertainties and the minimum measurable pressure help to optimize the experimental set up. Acoustic pressure estimations performed with LDV are compared with acoustic pressures obtained with a reference microphone. Measurements lead to a minimum bias of $0.006 \mathrm{~dB}$ and a minimum uncertainty of $0.013 \mathrm{~dB}$ on the acoustic pressure estimation for frequencies $1360 \mathrm{~Hz}$ and $680 \mathrm{~Hz}$.
\end{abstract}

Key words: microphone calibration, LDV, PIV, CRB, uncertainties, pressure estimation PACS: 43.38.Kb, 43.58. Vb, 43.60.Qv

\footnotetext{
* Corresponding author.

Email addresses: anne.degrooteuniv-lemans.fr(A. Degroot), rob.macdonaldeed.ac.uk (R. MacDonald),

olivier.richoux@univ-lemans.fr(O. Richoux),

bruno.gazengel@univ-lemans.fr(B. Gazengel ), dmc@ph.ed.ac.uk (M.

Campbell).
} 


\section{Notations}

Acoustics

$k \quad$ wavenumber

$\rho \quad$ air density at rest

$c \quad$ speed of sound at rest

$f \quad$ acoustic frequency

$\omega \quad$ acoustic angular frequency

$v(x, t) \quad$ acoustic particle velocity

$V(x) \quad$ acoustic particle velocity amplitude

$\Phi_{v} \quad$ acoustic particle velocity phase

$p(x, t) \quad$ acoustic pressure

$\bar{P}(x) \quad$ complex acoustic pressure amplitude

$P(x) \quad$ acoustic pressure amplitude

$\Phi_{p} \quad$ acoustic pressure phase

$\bar{R} \quad$ acoustic reflection coefficient in velocity

$R \quad$ acoustic reflection coefficient amplitude

$\Phi_{R} \quad$ acoustic reflection coefficient phase

$\bar{P}_{e}(x) \quad$ complex pressure amplitude at the rigid termination

$\bar{P}_{\text {ref }}(x) \quad$ reference pressure

\section{Optics}

$i \quad$ interfringe separation

$d_{x} \quad$ probe volume length

$\overline{I F}(x) \quad$ complex amplitude $I F \mathrm{e}^{j \phi_{I F}}$ of the instantaneous frequency of the Doppler signal

$I F(x) \quad$ amplitude of the instantaneous frequency of the Doppler signal

$\Phi_{I F} \quad$ phase of the instantaneous frequency of the Doppler signal

\section{Geometry}

$$
\begin{array}{lr}
x_{1} & \text { position of measurement } 1 \\
x_{2} & \text { position of measurement } 2 \\
\Delta x=x_{2}-x_{1}
\end{array}
$$

\section{Signal processing}


$S N R \quad$ Doppler Signal to Noise Ratio

$C R B \quad$ Cramer Rao Bounds for Doppler signal model

$F_{s} \quad$ Sampling frequency

General

$\bar{x} \quad$ is a complex value

$j=\sqrt{-1}$

$v_{p}(x, t) \quad$ particle velocity

$V_{f} \quad$ mean flow velocity amplitude 


\section{Introduction}

Measurement microphones are currently used for enclosed and free field applications. The accurate estimation of the measured pressure level requires a precise estimate of the microphone sensitivity. The sensitivity can be obtained in an absolute or in a relative manner. The absolute measurement of condenser microphone sensitivity provides primary calibration in metrology laboratories around the world. The relative calibration technique is used to estimate the sensitivity of typical microphones used in industrial or research applications.

For relative calibration, the sensitivity of the microphone is deduced from the sensitivity of a reference microphone measuring the same pressure amplitude as the microphone under test. In the case of absolute calibration, the sensitivity of the microphone under test is estimated without using a reference microphone. For both methods the calibration can be performed on pressure and free field microphones. These two types of microphone are fundamentally similar. However, pressure microphones are designed to measure sound pressure in a field where the sound pressure has the same magnitude and phase throughout, whereas free field microphones are designed to measure sound pressure in a field where sound waves propagate freely without obstruction.

Absolute calibration can be performed by implementing the reciprocity technique [1]. This technique is based on fine modelling of the physical effects involved in the measurement process. With the reciprocity technique three microphones (A,B and $\mathrm{C}$ ) are necessary. They are pair-wise coupled together (AB, AC, BC) by air (cavity for pressure microphone or free field for free field microphone). One of the two microphones under test emits sound while the second is used as a receiver. The electrical transfer impedance is calculated as the ratio of the receiver output voltage and the transmitter input current. The physical model of the transfer impedance between the input voltage and the output current enables estimation of the product of the two sensitivities. Repeating the process enables the three sensitivities to be deduced. Absolute calibration techniques can be viewed as a type of relative technique, the reference value being estimated by means of a physical model.

The reciprocity technique is commonly applied in enclosed field to estimate the sensitivity of a pressure microphone. This technique uses a small cavity and a good accuracy can be obtained in the sensitivity estimation, typically $\pm 0.05 \mathrm{~dB}$. The reciprocity calibration technique of microphones is approved by the International Electrotechnical Comission (IEC) and detailed in [1]. Although this method has been standardized, different authors are interested in increasing the measurement accuracy of the technique $[2,3]$ by developing more detailed modelling of the transfer impedance.

Free field microphone absolute calibration using the reciprocity technique has been 
studied by different authors [4,5] and has finally been standardized [6]. The absolute calibration of microphones in free field differs from the calibration of pressure microphones. In this case, the acoustic level produced by the emitting microphone is very low and the Signal to Noise Ratio (SNR) is small. This technique also requires that both microphones be considered as point sources located at some effective distance from the membrane (the acoustic centre). The sensitivity measurement needs to estimate the locations of these acoustic centres.

Free field calibration suffers from additional problems. If the microphone distance is sufficiently short, a standing wave can appear between the two membranes. The imperfect performance of the anechoic chamber that is used to provide the free field conditions can also generate reflections which can affect the transfer impedance estimation: it is necessary to use specific signal processing techniques to clean up the measured transfer impedance [4,5]. As a result of these problems, it would be useful to be able to calibrate free field microphones using non-intrusive techniques which would avoid the use of a second microphone as a sound source.

Optical methods such as Laser Doppler Velocimetry (LDV) or Particle Image Velocimetry (PIV) are non-intrusive measurement techniques which enable the measurement of acoustic velocity. LDV has been used for measuring low amplitude velocities since 1976 [7] while PIV has been used for measuring higher levels [8,9]. LDV provides a local measurement with good time resolution while PIV provides an estimate of the shape of a velocity field in a measuring area defined by the optical system. LDV is more precise than PIV, especially for measuring low velocity amplitudes, typically less than $1 \mathrm{~mm} / \mathrm{s}$. The main drawbacks of these two techniques are the complexity of the optical system and signal processing, and the high cost of experimental systems.

Different authors have proposed calibrating a pressure microphone using LDV. The microphone is located at the end of a tube attached to a loudspeaker and a physical model enables the deduction of the acoustic pressure from a single velocity measurement. Taylor [10] uses a spectral analysis of the Doppler signal delivered by the LDV system. He shows that it is possible to calibrate a condenser microphone with an accuracy of $\pm 0.03 \mathrm{~dB}$ when taking a large sample of measurements, resulting in a long measurement time. MacGillivray et al $[11,12]$ use the same approach to estimate the acoustic velocity with two methods, frequency analysis and Photon Correlation Spectroscopy. They show that the uncertainty in the pressure estimation is $\pm 0.1 \mathrm{~dB}$ when using spectral analysis and is $\pm 0.2 \mathrm{~dB}$ when using Photon Correlation Spectroscopy.

The issue of free field microphone calibration is clearly problematic. Both LDV and PIV have been used for characterising the acoustic velocity in a free field. Different authors $[13,14]$ show that the acoustic velocity can be measured with 
LDV. Gazengel et al. [15] show that LDV can measure the acoustic velocity profile on the axis of a radiating loudspeaker. PIV has been used by Degroot et al [16] for characterising the velocity field in the vicinity of the membrane of a microphone placed in a semi free field (Fig. 1).

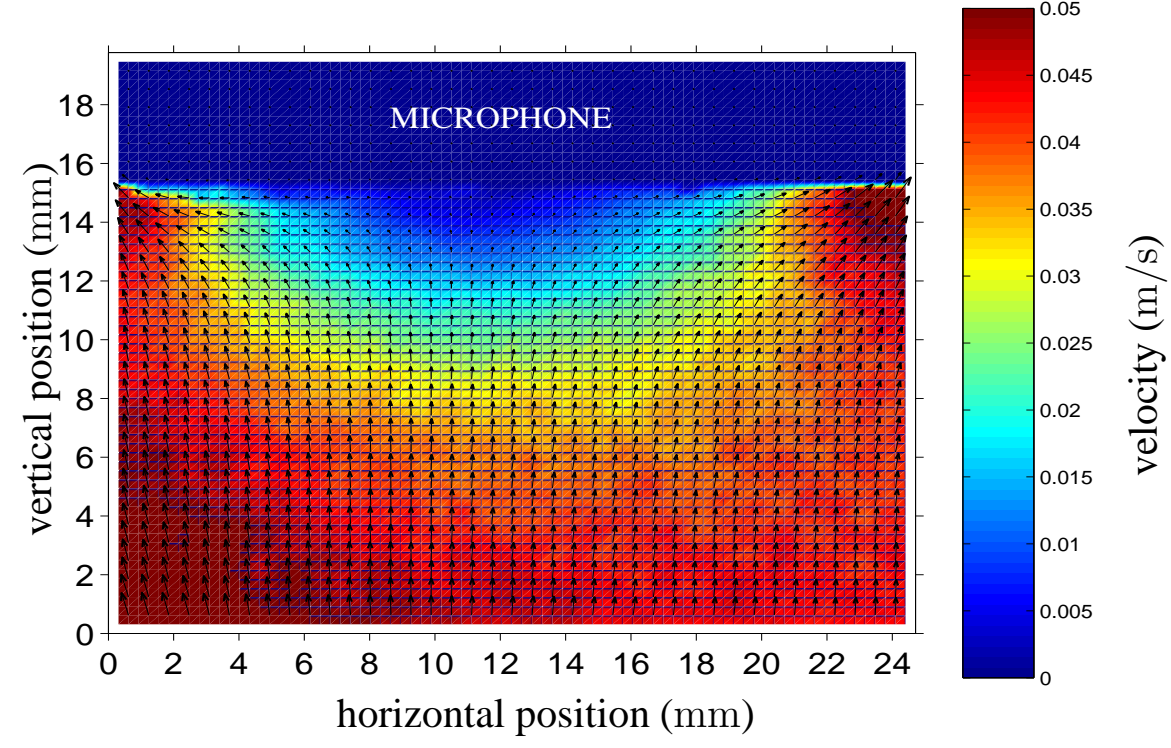

Figure 1. View of the velocity field in front a one inch microphone membrane measured with PIV [16].

However, these studies only deal with the measurement of acoustic velocity and do not propose to estimate the acoustic pressure. The calibration of microphones using optical non-intrusive techniques such as LDV or PIV requires the construction of a physical model of the acoustic field near the microphone membrane. Fig. (1) shows that a model with at least two dimensions should be developed for calibrating a free field microphone.

In this paper, as a first step towards being able to calibrate a free field microphone, we develop a new one dimensional physical model of the acoustic field near a microphone membrane. Building on the work of Taylor [7] and MacGillivray et al $[11,12]$, where the acoustic pressure was deduced from a single velocity measurement, the new model provides an estimate of the mean acoustic pressure acting on the microphone membrane from velocities measured at two different points located nearby. The new calibration method is tested on a pressure microphone in an enclosed field.

In the next section, the analytical model which yields an estimation of the acoustic pressure in the waveguide using two acoustic velocity measurements is presented. Moreover, the uncertainties in the pressure amplitude and phase are derived using the theoretical minimum uncertainty in the LDV velocity measurement.

In Section 3, the details of the experimental study are presented. First the experi- 
mental system used for assessing the calibration method is described and then the uncertainty analysis developed in Section 2 is applied to the design of the experiment. Finally, experimental results are presented and discussed.

\section{Pressure estimation model}

\subsection{Pressure calculation}

Consider an incident plane wave with acoustic particle velocity amplitude $V_{0}$ and wavenumber $k$ that experiences viscothermal losses [17] as it propagates from left to right along the $\mathrm{x}$-axis in a waveguide having a reflection coefficient $\bar{R}=R e^{j \Phi_{R}}$ (see Fig. 2).

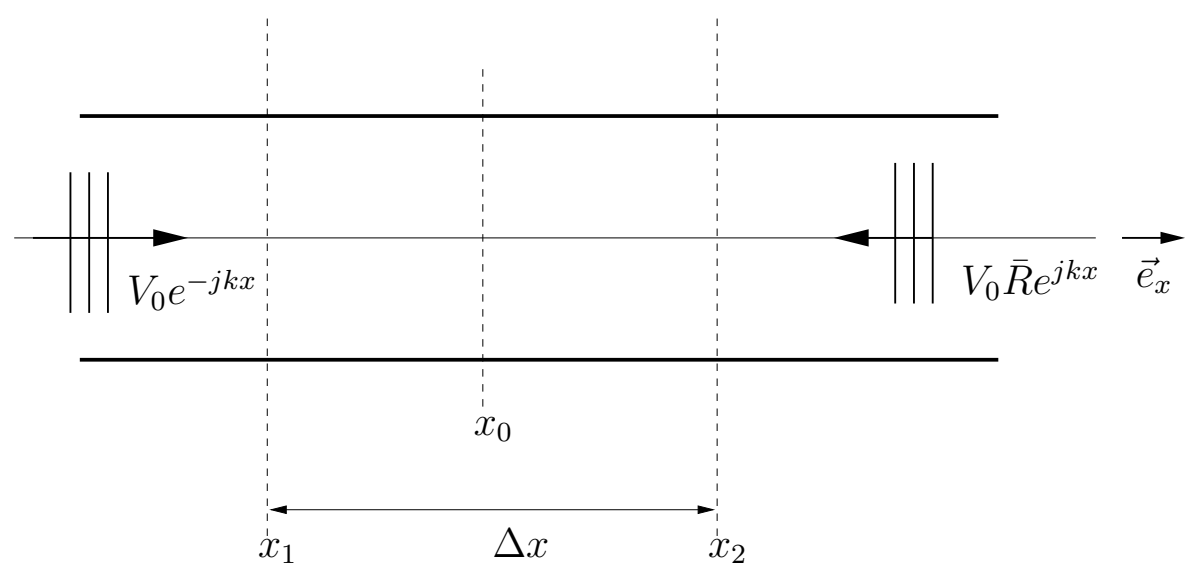

Figure 2. Schematic view of the problem

The mass conservation law gives an expression relating the acoustic pressure $p(x, t)$ and the acoustic particle velocity $\vec{v}(x, t)$

$$
\frac{\partial p(x, t)}{\partial t}=-\rho c^{2} \operatorname{div}(\vec{v}(x, t))
$$

where $\rho$ is the air density at rest and $c$ is the speed of sound. In the case of a harmonic plane wave of angular frequency $\omega$ propagating along the $x$ axis, acoustic pressure and particle velocity can be written as

$$
\begin{aligned}
& \vec{v}(x, t)=\bar{V}(x) e^{j \omega t} \vec{e}_{x}=V(x) e^{j \Phi_{v}(x)} e^{j \omega t} \vec{e}_{x}, \\
& p(x, t)=\bar{P}(x) e^{j \omega t}=P(x) e^{j \Phi_{p}(x)} e^{j \omega t} .
\end{aligned}
$$

where $V(x), P(x), \Phi_{v}(x)$ and $\Phi_{p}(x)$ are respectively the acoustic velocity amplitude, acoustic pressure amplitude, phase of acoustic velocity and phase of acoustic pressure at abscissa $x$. 
Using Eqs. 1, 2 and 3,

$$
j \omega \bar{P}(x)=-\rho c^{2} \frac{d \bar{V}(x)}{d x} .
$$

Knowing that the acoustic velocity is written

$$
\bar{V}(x)=V_{0}\left(e^{-j k x}+\bar{R} e^{j k x}\right),
$$

the spatial derivative of the velocity at abscissa $x_{0}$ is given by [18]

$$
\left.\frac{d \bar{V}}{d x}\right|_{x_{0}}=\frac{\Delta \bar{V}}{\Delta x} \frac{1}{\operatorname{sinc}\left(\frac{k \Delta x}{2}\right)}
$$

where $\Delta \bar{V}=\bar{V}\left(x_{2}\right)-\bar{V}\left(x_{1}\right)$ with $x_{1}=x_{0}-\Delta x / 2$ and $x_{2}=x_{0}+\Delta x / 2$ (see Fig. 2).

Using Eqs. 4 and 6, the acoustic pressure at abscissa $x_{0}$ is given by

$$
\bar{P}\left(x_{0}\right)=j \rho c \frac{\Delta \bar{V}}{2 \sin \left(\frac{k \Delta x}{2}\right)} .
$$

\subsection{Minimum uncertainty in the pressure estimation}

\subsubsection{General formulation}

This section aims to determine the minimum uncertainty in the pressure modulus and phase using the uncertainties in the acoustic velocity measured by means of LDV and the uncertainties in the physical quantities $(\rho, c, \Delta x)$.

The relative uncertainty $\frac{\delta P\left(x_{0}\right)}{P\left(x_{0}\right)}$ in the estimation of the amplitude pressure at $x=x_{0}$ is obtained using the Eq. 7 and is written

$$
\frac{\delta P\left(x_{0}\right)}{P\left(x_{0}\right)}=\left[\left(\frac{\delta \Delta V}{\Delta V}\right)^{2}+\left(\frac{\delta(k \Delta x)}{2|\tan (k \Delta x / 2)|}\right)^{2}+\left(\frac{\delta c}{c}\right)^{2}+\left(\frac{\delta \rho}{\rho}\right)^{2}\right]^{1 / 2},
$$

where $\Delta V=|\Delta \bar{V}|, \delta \rho / \rho$ is the relative uncertainty in the density, $\delta c / c=-\delta \rho / 2 \rho$ is the relative uncertainty in the speed of sound, assuming that the speed of sound only depends on the density $\rho$ considered as an equivalent density as defined in [19]. The uncertainty in $k \Delta x$ is defined by [19]

$$
\delta(k \Delta x)=k \Delta x\left[\left(\frac{\delta \rho}{2 \rho}\right)^{2}+\left(\frac{\delta \Delta x}{\Delta x}\right)^{2}\right]^{1 / 2} .
$$


In the same way, the uncertainty in the phase of the pressure can be obtained using Eq. 7

$$
\delta \Phi_{p}=\delta \Phi_{\Delta \bar{V}}
$$

where $\Phi_{\Delta \bar{V}}$ is the phase of $\Delta \bar{V}$.

\subsubsection{Minimum uncertainty in the velocity difference}

The determination of the minimum uncertainty in the acoustic pressure (Eqs. 8 and 10) requires the calculation of the minimum uncertainty in the velocity difference. Using the notation $V_{1}=V\left(x_{1}\right), V_{2}=V\left(x_{2}\right), \Phi_{1}=\Phi_{v}\left(x_{1}\right)$ and $\Phi_{2}=\Phi_{v}\left(x_{2}\right)$, the relative uncertainty in $\Delta V$ is

$$
\begin{aligned}
\frac{\delta \Delta V}{\Delta V}= & \frac{1}{(\Delta V)^{2}}\left[\left(\delta V_{2}\right)^{2}\left[V_{2}-V_{1} \cos (\Delta \Phi)\right]^{2}+\left(\delta V_{1}\right)^{2}\left[V_{1}-V_{2} \cos (\Delta \Phi)\right]^{2}\right. \\
& \left.+\left[V_{1} V_{2} \sin (\Delta \Phi)\right]^{2}\left[\left(\delta \Phi_{1}\right)^{2}+\left(\delta \Phi_{2}\right)^{2}\right]\right]^{1 / 2},
\end{aligned}
$$

where $\Delta \Phi=\Phi_{2}-\Phi_{1}$ and $\delta V_{1}, \delta V_{2}, \delta \Phi_{1}$ and $\delta \Phi_{2}$ are respectively the uncertainties in velocity amplitude and phase at abscissa $x_{1}$ and $x_{2}$.

The uncertainty in the phase of the velocity difference $\Phi_{\Delta \bar{V}}$ is

$$
\begin{aligned}
\delta \Phi_{\Delta \bar{V}}= & \frac{1}{(\Delta V)^{2}}\left[\left(\delta V_{1}\right)^{2} V_{2}^{2} \sin ^{2}(\Delta \Phi)+\left(\delta \Phi_{1}\right)^{2}\left[V_{1}^{2}-V_{1} V_{2} \cos (\Delta \Phi)\right]^{2}\right. \\
& \left.+\left(\delta V_{2}\right)^{2} V_{1}^{2} \sin ^{2}(\Delta \Phi)+\left(\delta \Phi_{2}\right)^{2}\left[V_{2}^{2}-V_{1} V_{2} \cos (\Delta \Phi)\right]^{2}\right]^{1 / 2}
\end{aligned}
$$

The particle velocity $v_{p}(x, t)$ measured by LDV at abscissa $x$ can be estimated using [20]

$$
v_{p}(x, t)=i \cdot \overline{I F}(x, t),
$$

where $i$ is the interfringe separation of the LDV probe and

$$
\overline{I F}(x, t)=I F(x) \cdot e^{j \phi_{I F(x)}} \cdot e^{j \omega t}
$$

is the complex instantaneous frequency defined by [21]

$$
\overline{I F}(x, t)=\frac{1}{2 \pi} \frac{d \Phi_{D}(x, t)}{d t}
$$

where $\Phi_{D}(x, t)$ is the phase of the LDV probe signal, proportional to the particle displacement $x_{p}(t)$ in the probe volume $\left(\Phi_{D}(x, t)=\frac{2 \pi}{i} x_{p}(t)\right)$. In the case of sinusoïdal acoustic excitation, $\overline{I F(x, t)}$ is estimated by specific signal processing ( Short Time Fourier Transform [22], Cross Wigner-Ville distribution [23,24]). The amplitude $I F(x)$ and phase $\phi_{I F(x)}$ are estimated with a Least Mean Square Method 
[25]. The uncertainties in the acoustic velocity amplitude $V$ and phase $\Phi_{v}$ measured by LDV are given by

$$
\begin{aligned}
\frac{\delta V}{V} & =\sqrt{\left(\frac{\delta i}{i}\right)^{2}+\left(\frac{\delta I F}{I F}\right)^{2}}, \\
\delta \Phi_{v} & =\delta \Phi_{I F},
\end{aligned}
$$

where $\frac{\delta i}{i}$ is the relative uncertainty in the interfringe separation measurement and $\frac{\delta I F}{I F}$ is the relative uncertainty in the instantaneous frequency amplitude estimation. The minimum uncertainty in the instantaneous frequency is given by the minimum variance in the estimation of the acoustic parameters $V(x)$ and $\Phi_{v}(x)$. This minimum variance, depending on the signal, is expressed by the Cramer-Rao Bounds (CRB), estimated using a model of the Doppler signal [26] . In the case of harmonic excitation at acoustic frequency $f$ and for low mean flow velocity encountered in an enclosed field (typically 0.5 to $2 \mathrm{~mm} / \mathrm{s}$ due to thermal effects generated by the loudspeaker), Le Duff et al [26] give the CRB for the acoustic velocity amplitude $V(x)$ and phase $\Phi_{v}(x)$

$$
\begin{aligned}
& C R B(V)=2 \sqrt{\frac{2}{\pi}} \frac{F}{\alpha^{2} N_{p} \mathrm{SNR}} V^{2}, \\
& C R B\left(\Phi_{v}\right)=2 \sqrt{\frac{2}{\pi}} \frac{F}{\alpha^{2} N_{p} \mathrm{SNR}},
\end{aligned}
$$

with

$$
\begin{aligned}
N_{p} & =\frac{2 f d_{x}}{V_{f}}, \\
F & =\frac{f}{F_{s}}, \\
\alpha & =\frac{V}{i f},
\end{aligned}
$$

where $V_{f}$ is the mean flow velocity, $F_{s}$ is the sampling frequency and $d_{x}$ is the length of the probe volume along the $x$ axis [26].

Using Eqs. 11 and 18, the minimum relative uncertainty in the difference in velocity amplitude can be written

$$
\left.\frac{\delta \Delta V}{\Delta V}\right|_{\min }=\frac{2 \sqrt{2} K i f}{\Delta V}\left[1+\left(\frac{\delta i}{i}\right)^{2} \frac{\left(V_{1}^{2}-V_{1} V_{2} \cos \Delta \Phi\right)^{2}+\left(V_{2}^{2}-V_{1} V_{2} \cos \Delta \Phi\right)^{2}}{2 \Delta V^{2}(2 K i f)^{2}}\right]^{1 / 2}
$$


where

$$
K=\sqrt{2 \sqrt{\frac{2}{\pi}} \frac{F}{N_{p} \mathrm{SNR}}} .
$$

Considering that the acoustic particle velocity is written as the sum of an outgoing and incoming wave (Eq. 5), the minimum relative uncertainty in the difference in velocity amplitude can be obtained using

$$
\Delta V=2 V_{0}\left|\sin \frac{k \Delta x}{2}\right|\left|e^{-j k x_{0}}-R e^{j \phi_{R}} e^{j k x_{0}}\right|,
$$

in Eq. 23. The uncertainty in the phase of the velocity difference is

$$
\left.\delta \Phi_{\Delta \bar{V}}\right|_{\min }=\frac{2 \sqrt{2} K i f}{\Delta V}
$$

Eqs. 23 and 26 show that the uncertainty in the velocity difference (amplitude and phase) is minimized for $\Delta V$ large, which occurs when measuring two velocities having the same amplitude and opposite phase.

\subsubsection{Total minimum uncertainty in the pressure}

The total relative uncertainty in the pressure amplitude estimation is found by substituting Eqs. 23 and 25 into Eq. 8

$$
\begin{aligned}
\left.\frac{\delta P\left(x_{0}\right)}{P\left(x_{0}\right)}\right|_{\min }= & {\left[\left(\frac{2 \sqrt{2} K i f}{\Delta V}\left[1+\left(\frac{\delta i}{i}\right)^{2} \frac{\left(V_{1}^{2}-V_{1} V_{2} \cos \Delta \Phi\right)^{2}+\left(V_{2}^{2}-V_{1} V_{2} \cos \Delta \Phi\right)^{2}}{2 \Delta V^{2}(2 K i f)^{2}}\right]^{1 / 2}\right)^{2}\right.} \\
& \left.+\left(\frac{\delta(k \Delta x)}{2\left|\tan \frac{k \Delta x}{2}\right|}\right)^{2}+\frac{5}{4}\left(\frac{\delta \rho}{\rho}\right)^{2}\right]^{1 / 2}
\end{aligned}
$$

with $K$ given in Eq. 24.

The uncertainty in the pressure phase is given by

$$
\left.\delta \Phi_{p}\right|_{\min }=\left.\delta \Phi_{\Delta \bar{V}}\right|_{\min },
$$

where $\left.\delta \Phi_{\Delta \bar{V}}\right|_{\text {min }}$ is given in Eq. 26 . 


\subsection{Minimum measurable pressure}

The minimum acoustic pressure amplitude that can be estimated using this method is given by Eq. (7)

$$
P\left(x_{0}\right)_{\text {min }}=\rho c \frac{\Delta V_{\text {min }}}{\left|2 \sin \left(\frac{k \Delta x}{2}\right)\right|},
$$

where $\Delta V_{\min }$ is the minimum measurable amplitude of the velocity difference.

\section{Experimental study}

\subsection{Experimental system}

The experimental study aims at determining the bias and standard deviation of the pressure estimated using two velocity measurements and Eq. (7). For this, the acoustic pressure is estimated inside a tube excited with a loudspeaker at one end and closed by a rigid termination at the other end. The estimated pressure is compared with a reference pressure obtained by means of a microphone flush-mounted at the end of the tube.

\subsubsection{Acoustic system}

The acoustic set-up consists of a square cross-section duct $\left(0.1 \times 0.1 \mathrm{~m}^{2}\right)$ made of Perspex of thickness $10 \mathrm{~mm}$. The cut-off frequency associated with the first transverse acoustic mode is $1720 \mathrm{~Hz}$. The tube is $0.5 \mathrm{~m}$ long. A loudspeaker (GUO GUANG 450100) is mounted at one end of the waveguide and the other end is closed by a rigid termination (see Fig. 3). The acoustic pressure is measured at the rigid end of the tube by means of a $1 / 2$ inch microphone (B\&K 4192) connected to a preamplifier (B\&K 2619) and a conditioning amplifier (B\&K 2609).

\subsubsection{LDV system}

The LDV apparatus used in this study is a dual beam system operating in the differential Doppler mode. Only one velocity component is measured. The laser source, producing light of $514.5 \mathrm{~nm}$ wavelength in air, is installed in a separate room with the beams delivered to the lab using fibre optics. In order to get enough light intensity the forward scattering configuration is used. The laser power is $20 \mathrm{~mW}$ at the location of the probe volume. The focal distance of the emitting optics is 1000 $\mathrm{mm}$. The angle between the two incident beams is set to about $28 \mathrm{deg}$. The probe volume length $d_{x} \simeq 0.1 \mathrm{~mm}$ and the interference pattern contains about 100 fringes. 


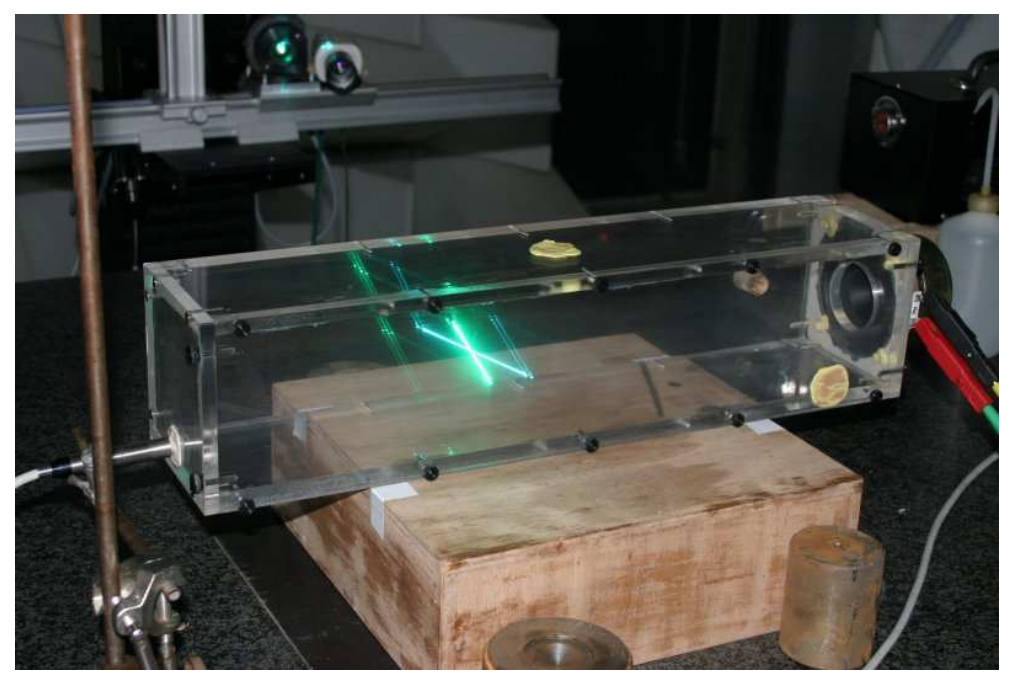

Figure 3. View of the acoustic system.

The emitting and receiving optics are supported by a traverse system which enables to measure the velocity at different locations. The velocity sign is determined using a Bragg cell introduced in the path of one of the incident beams. The Bragg cell operates here in the -1 mode, which decreases the frequency value of the beam that is frequency shifted. The cell is driven at $f_{B}=40 \mathrm{MHz}$. The seeding is SAFEX Super Fog Fluid, injected inside the tube.

The optical signal is converted into an electrical signal by means of a photomultiplier (PM) using a $1200 \mathrm{~V}$ supply. The signal is converted into two signals in quadrature and analysed using the Phase Derivative Based Estimator [26] in order to estimate the instantaneous frequency. The parameters of the acoustic velocity $\left(V, \Phi_{v}\right)$ are calculated using a Least Mean Square Method [25] applied to the instantaneous frequency defined in section 2.2.2.

The acquisition of Doppler signals was performed using $F_{s}=1 \mathrm{MHz}$ and $F_{s}=$ $500 \mathrm{kHz}$. The velocities measured at abscissa $x_{1}$ and $x_{2}$ were estimated using 10 acquisitions, with each signal comprising of about 10 bursts. This enables 10 values of the acoustic parameters $V$ and $\Phi_{v}$ to be estimated. The mean of these 10 gives the estimation of acoustic velocity and the standard deviations $\sigma_{V}$ and $\sigma_{\Phi_{v}}$. The uncertainties are then $\delta_{V}=\frac{2 \sigma_{V}}{\sqrt{10}}$ and $\delta_{\Phi_{v}}=\frac{2 \sigma_{\Phi_{v}}}{\sqrt{10}}$.

\subsection{Experiment design}

The aim of this section is to define an experimental configuration which minimizes the uncertainty in the pressure estimation. By assuming that the termination is rigid $(\bar{R}=-1)$, the minimum measurable pressure is calculated (see Eq. 33). Then, in section 3.2.2, the uncertainties in the pressure estimated with LDV and with the reference microphone are studied. We use parameters encountered in the usual 
experimental configuration as given in table 1.

Table 1

\begin{tabular}{cccccccc}
\hline$\frac{\delta \rho}{\rho}$ & $\frac{\delta i}{i}$ & $\delta L$ & $\mathrm{i}$ & $F_{s}$ & $\mathrm{SNR}$ & $V_{f}$ & $d_{x}$ \\
\hline $0.05 \%$ & $0.1 \%$ & $0.1 \mathrm{~mm}$ & $1 \mu \mathrm{m}$ & $1 \mathrm{MHz}$ & $15 \mathrm{~dB}$ & $5 \mathrm{~mm} / \mathrm{s}$ & $0.1 \mathrm{~mm}$ \\
\hline
\end{tabular}

Values of parameters corresponding to usual experimental configuration in enclosed field.

Eqs. 27 and 28, which give the uncertainty in the amplitude and phase of the pressure estimated with LDV, are made up of three terms and one term respectively. The last term of Eq. 27 is a constant value describing the uncertainty in the air density due to the seeding. The second term of Eq. 27 shows the effect of the microphone probe spacing (term in $k \Delta x$ ). The first term of Eq. 27 shows the uncertainty in the acoustic velocity estimation due to the LDV signal processing (term in Kif) and to the interfringe measurement (term in $\delta i$ ). Eq. 28 shows that the uncertainty in the phase depends only on the signal processing (term in Kif). For $\bar{R}=-1$, the uncertainty in the velocity estimation is minimized using $k x_{0}=m \pi(m \in \mathbb{N})$, which means that the pressure is best estimated at one of its antinodes. This configuration is used in the experiment. Assuming that the velocity is measured at

$$
\begin{aligned}
& x_{1}=x_{0}+\frac{\Delta x}{2} \text { and } \\
& x_{2}=x_{0}-\frac{\Delta x}{2},
\end{aligned}
$$

$\bar{V}_{1}=-\bar{V}_{2}$ if $\Delta x$ is chosen correctly, which leads to $V_{1}=V_{2}$ and $\Delta \phi=\pi$. These assumptions enable the deduction of the uncertainties in the amplitude and phase of the pressure estimated with LDV.

\subsubsection{Minimum measurable pressure}

Using the assumption $\bar{V}_{1}=-\bar{V}_{2}$, the minimum measurable amplitude of the velocity difference is written $\Delta V_{\text {min }}=\delta V_{1}+\delta V_{2}$. Using Eq. 16, Eq. 18 and Eq. 22, $\Delta V_{\min }$ is given by

$$
\Delta V_{\text {min }}=2 K i f\left[\left(1+\frac{(\delta i / i)^{2}}{\left(\frac{2 K i f}{V_{1}}\right)^{2}}\right)^{1 / 2}+\left(1+\frac{(\delta i / i)^{2}}{\left(\frac{2 K i f}{V_{2}}\right)^{2}}\right)^{1 / 2}\right]
$$

The minimum measurable pressure is given by Eq. (29) and takes the following form

$$
P\left(x_{0}\right)_{\min }=\rho c \frac{2 K i f}{\left|\sin \frac{k \Delta x}{2}\right|}\left[1+\left(\frac{\delta i}{i}\right)^{2}\left(\frac{V_{0} \sin \frac{\Delta x}{2}}{K i f}\right)^{2}\right]^{1 / 2} \text {. }
$$


Considering the acoustic field in a resonant tube and using the conservation of mass law $P\left(x_{0}\right)=2 \rho c V_{0}\left|\cos k x_{0}\right|$, the minimum pressure is

$$
P\left(x_{0}\right)_{\min }=\frac{2 \rho c K i f}{\left|\sin \frac{k \Delta x}{2}\right|} \frac{1}{\sqrt{1-\left(\frac{\delta i}{i}\right)^{2}}} .
$$

Fig. 4 shows the minimum pressure amplitude that can be estimated using two LDV measured velocities as a function of frequency for different spacings $\Delta x$ and using parameters given in table 1 .

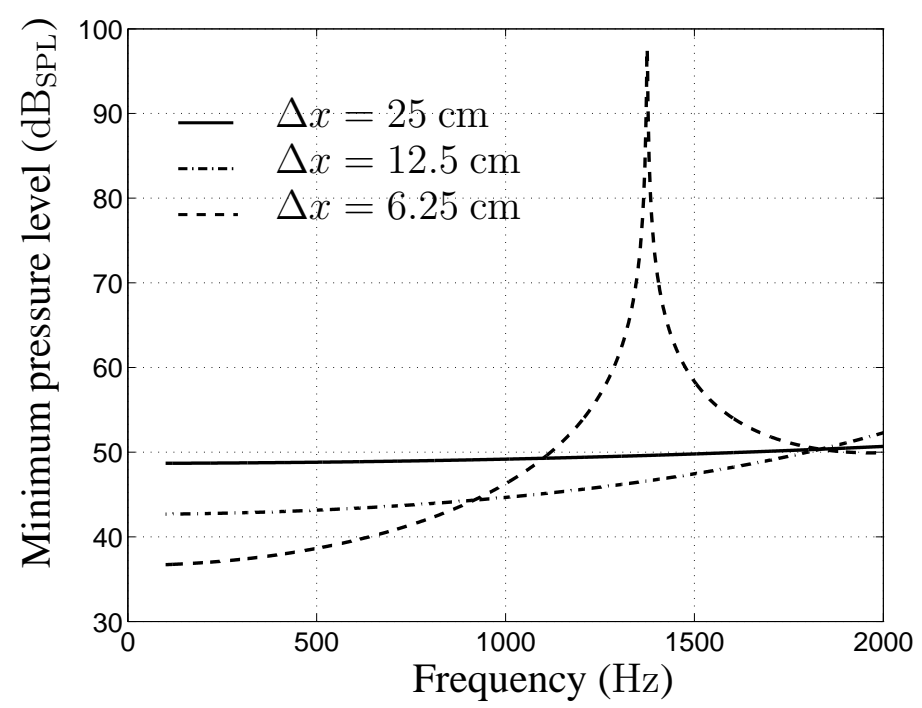

Figure 4. Minimum measurable pressure level with LDV as a function of the acoustic frequency $f$ for different probe spacings $\Delta x(-25 \mathrm{~cm},-\cdot-12.5 \mathrm{~cm}$ and $--6.25 \mathrm{~cm})$

\subsubsection{Uncertainty in the pressure estimation}

LDV estimated pressure Eq. 30 and the condition $k x_{0}=\pi$ combined with Eq. 25 lead to

$$
\Delta V=4 V_{0}\left|\sin \frac{k \Delta x}{2}\right|
$$

Eq. 30, Eq. 31 and the condition $k x_{0}=\pi$ combined with Eq. 5 lead to

$$
V_{1} V_{2}=2 V_{0}^{2}|1-\cos k \Delta x|
$$

The total minimum relative uncertainty in the pressure amplitude estimation is 


$$
\begin{array}{r}
\left.\frac{\delta P\left(x_{0}\right)}{P\left(x_{0}\right)}\right|_{\min }=\left[\left(\frac{\sqrt{2} K i f}{2 V_{0}\left|\sin \frac{k \Delta x}{2}\right|}\right)^{2}\left[1+\left(\frac{\delta i}{i}\right)^{2} \frac{V_{0}^{2}|1-\cos k \Delta x|}{(2 K i f)^{2}}\right]\right. \\
\left.+\left(\frac{\delta(k \Delta x)}{2 \mid \tan \left(\frac{k \Delta x}{2} \mid\right)}\right)^{2}+\frac{5}{4}\left(\frac{\delta \rho}{\rho}\right)^{2}\right]^{1 / 2}
\end{array}
$$

and the total minimum uncertainty in the pressure phase estimation is

$$
\left.\delta \Phi_{p}\right|_{\min }=\frac{\sqrt{2} K i f}{2 V_{0}\left|\sin \frac{k \Delta x}{2}\right|} .
$$

In order to minimize the uncertainties given in Eqs. (37) and (38), the probe spacing $\Delta x$ should satisfy $\frac{k \Delta x}{2}=(2 n+1) \frac{\pi}{2}(n \in N)$, which corresponds to $\Delta x=(2 n+$ 1) $\lambda / 2, \lambda$ being the acoustic wavelength. However this spacing can be very large for low frequencies and such configurations can be unusable. For this reason, different values of $\Delta x$ are used in the experiment. Eqs. 37, 38 show that the uncertainty is minimized if the velocity amplitude is large. Excitation of the tube at the resonance frequency is therefore favourable. The uncertainties in the pressure amplitude and the pressure phase, estimated with LDV, are shown in Fig. 5 and 6 respectively for an acoustic frequency of $680 \mathrm{~Hz}$ and a spacing $\Delta x=6.25 \mathrm{~cm}$.

Microphone reference pressure The reference pressure $\bar{P}_{r e f}(x)$ is obtained using the pressure $\bar{P}_{e}$ measured by a microphone mounted flush at the end of the tube

$$
\bar{P}_{r e f}(x)=\bar{P}_{e} \cos (k x) \text {. }
$$

The relative uncertainty in the reference pressure amplitude at $x_{0}$ is given by

$$
\frac{\delta P_{r e f}\left(x_{0}\right)}{P_{r e f}\left(x_{0}\right)}=\left[\left(\frac{\delta P_{e}}{P_{e}}\right)^{2}+\left(\delta\left(k x_{0}\right) \tan k x_{0}\right)^{2}\right]^{1 / 2},
$$

with

$$
\frac{\delta P_{e}}{P_{e}}=\left[\left(\frac{\delta U_{c}}{U_{c}}\right)^{2}+\left(\frac{\delta U_{e}}{U_{e}}\right)^{2}+\left(\frac{\delta U_{f}}{U_{f}}\right)^{2}\right]^{1 / 2},
$$

where $\delta U_{c} / U_{c}$ is the relative uncertainty due to the microphone calibrator, $\delta U_{e}$ is the uncertainty in the measured voltage of the reference microphone due to variation of the air density during the measurement duration and $\delta U_{f}$ is the uncertainty in the calibration voltage $\left(\delta U_{f}=U_{\text {res }} / 3\right.$ with $U_{\text {res }}$ the resolution of the sinusoidal voltage signal). The relative uncertainty $\delta P_{r e f}\left(x_{0}\right) / P_{r e f}\left(x_{0}\right)$ equals $\delta P_{e} / P_{e}$ using the condition $k x_{0}=\pi$. 
Considering that the excitation frequency is a resonant frequency of the tube (680 $\mathrm{Hz}$ ), we choose values of $\Delta x$ which minimize the uncertainty in the pressure amplitude for an acoustic level of $90 \mathrm{~dB}_{\mathrm{SPL}}$ (see table 2).

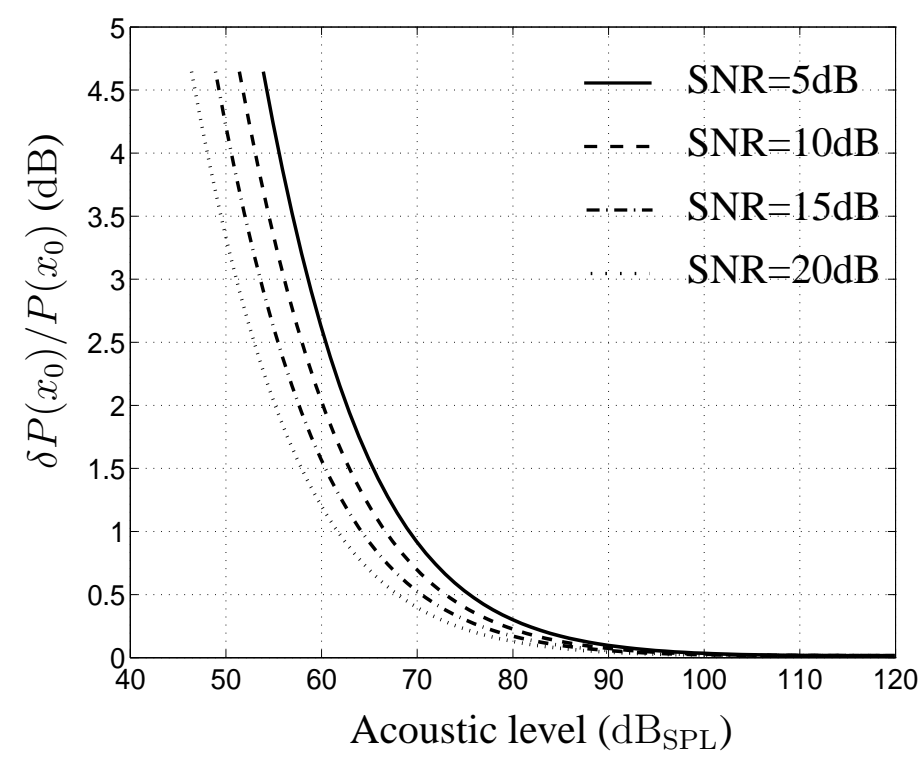

Figure 5. Uncertainty in the pressure amplitude estimated with LDV as a function of the acoustic level for an acoustic frequency of $680 \mathrm{~Hz}$ and a spacing $\Delta x=6.25 \mathrm{~cm}$ ($\mathrm{SNR}=5 \mathrm{~dB},--\mathrm{SNR}=10 \mathrm{~dB},-\cdot-\mathrm{SNR}=15 \mathrm{~dB}$ and $\cdots \mathrm{SNR}=20 \mathrm{~dB})$.

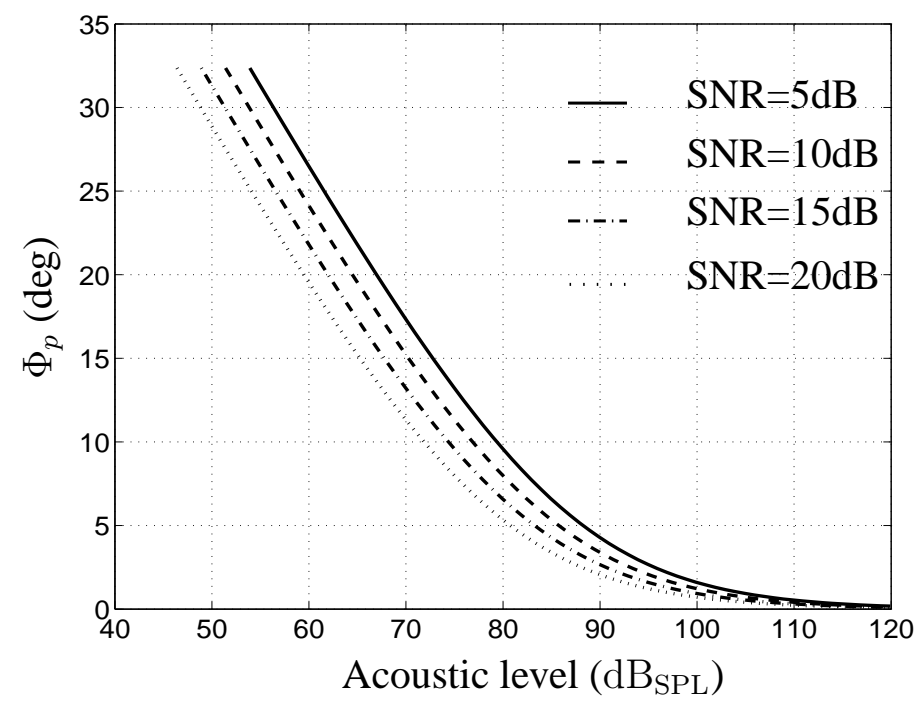

Figure 6. Uncertainty in the pressure phase estimated with LDV as a function of the acoustic level for an acoustic frequency of $680 \mathrm{~Hz}$ and a spacing $\Delta x=6.25 \mathrm{~cm}(-\mathrm{SNR}=5 \mathrm{~dB}$, $--\mathrm{SNR}=10 \mathrm{~dB},-\cdot-\mathrm{SNR}=15 \mathrm{~dB}$ and $\cdots \mathrm{SNR}=5 \mathrm{~dB})$.

For frequency $f=680 \mathrm{~Hz}$ and a probe spacing $\Delta x=6.25 \mathrm{~cm}$, Fig. 5 and Fig. 6 show the uncertainty in the pressure amplitude and phase estimation as a function of the acoustic level measured at the rigid end of the tube for different Doppler Signal to Noise Ratio. These figures show that for a SNR of $15 \mathrm{~dB}$, which is commonly encountered in LDV measurement, the minimum relative uncertainty in the pres- 
sure amplitude is less than $0.06 \mathrm{~dB}$ if the acoustic level is greater than $90 \mathrm{~dB} \mathrm{BPL}_{\mathrm{SPL}}$. The minimum uncertainty in the pressure phase is less than 2.6 degrees if the acoustic level is greater than $90 \mathrm{~dB}_{\mathrm{SPL}}$. These uncertainties are mainly due to the signal processing (first term in Eq. 37) especially when the acoustic level is low ( $90 \mathrm{~dB} \mathrm{BPL}_{\mathrm{SP}}$ : $3.5 \cdot 10^{-4} \mathrm{~dB}$ and $49 \mathrm{~dB}_{\mathrm{SPL}}: 3.52 \mathrm{~dB}$ for a $\mathrm{SNR}=15 \mathrm{~dB}$ ). The uncertainty associated with the second term of Eq. 37 does not depend on the acoustic level and is about $2 \cdot 10^{-5} \mathrm{~dB}$.

\subsection{Results}

Experimental results have been obtained using the conditions given in Table 2. In this experiment, the reference microphone was calibrated using a B\&K 4231 calibrator with accuracy $\pm 0.2 \mathrm{~dB}_{\mathrm{SPL}}$.

Table 2

\begin{tabular}{|c|c|c|c|c|c|c|}
\hline $\mathrm{f}(\mathrm{Hz})$ & $x_{0}\left(k x_{0}=\pi\right)(\mathrm{cm})$ & \multicolumn{2}{|c|}{ Level $\left(\mathrm{dB}_{\mathrm{SPL}}\right)$} & \multicolumn{3}{|c|}{$\Delta x(\mathrm{~cm})$} \\
\hline 680 & 25 & 90 & 120 & $6.25_{(1)}$ & $12.5_{(2)}$ & $25_{(3)}$ \\
\hline 1360 & 12.5 & 90 & 120 & $3.125_{(4)}$ & $6.25_{(5)}$ & $12.5_{(6)}$ \\
\hline
\end{tabular}

Values of parameters used for making the experiment in enclosed field.

Initially, the velocity pattern in the tube was measured at $f=680 \mathrm{~Hz}$ and at an acoustic level of $120 \mathrm{~dB}$ using LDV and compared with the velocity

$$
\bar{V}_{r e f}(x)=j \frac{\sin k x}{\rho c} \bar{P}_{e}
$$

estimated using pressure $\bar{P}_{e}$ measured at the end of the tube. Fig. 7 shows that LDV provides an estimate of the velocity pattern which is in good agreement with the reference velocity. However, a bias of $1.8 \mathrm{~mm} / \mathrm{s}$ ( $2.6 \%$ corresponding to 0.22 $\mathrm{dB}_{\mathrm{SPL}}$ ) can be seen between the two curves.

Secondly, the pressure at $x_{0}=\lambda / 2$ was estimated with LDV and compared with the reference pressure as shown in Fig. 8. For these experiments, the acoustic velocity was measured using a single LDV probe, moving between the two measurement points $x_{1}$ and $x_{2}$ with the traverse system (section 3.1.2). The measurement was repeated 10 times after the seeding was introduced into the tube. In this configuration, the acoustic pressure amplitude changes during the experiment because the air density varies with the seeding density. These variations serve to maximise the uncertainties in the reference and estimated pressures due to the finite time for the displacement of the traverse system between the two measurements. These uncertainties would be smaller with two simultaneous LDV measurements.

The observed bias values (mean of the difference between reference and estimated 


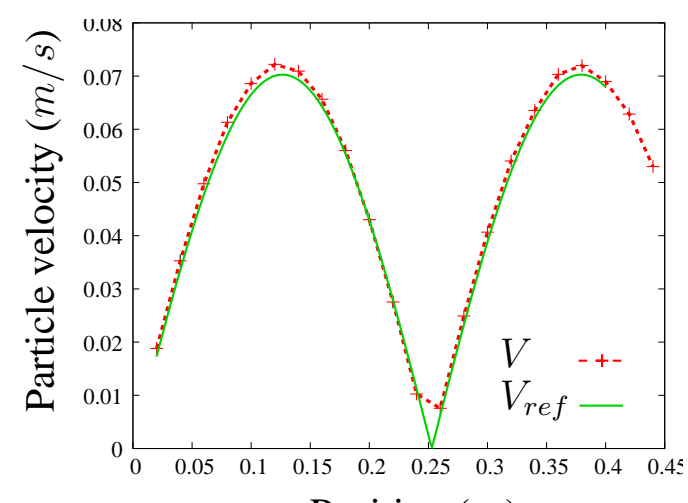

Position $(m)$

(a)

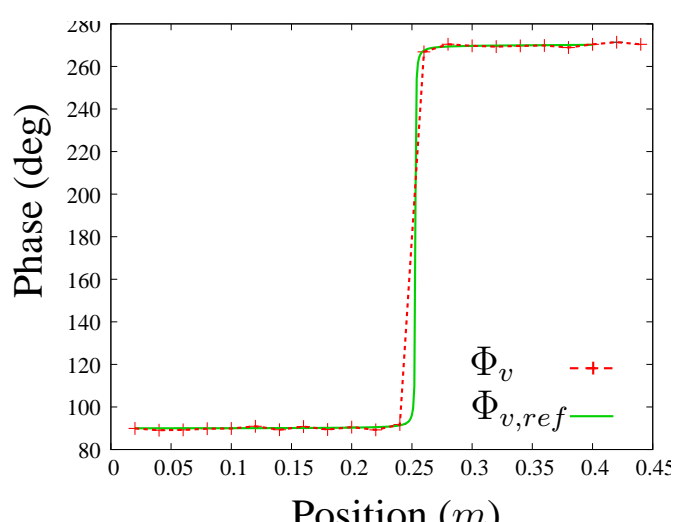

Position $(m)$

(b)

Figure 7. Velocity profile in the tube for an acoustic level of $120 \mathrm{~dB}_{\mathrm{SPL}}$ and an acoustic frequency of $680 \mathrm{~Hz}$.

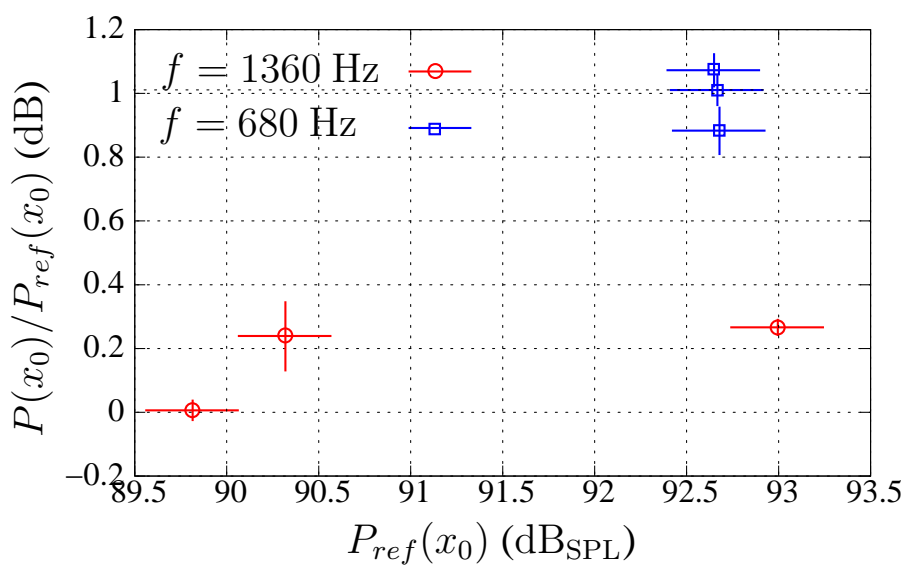

(a)

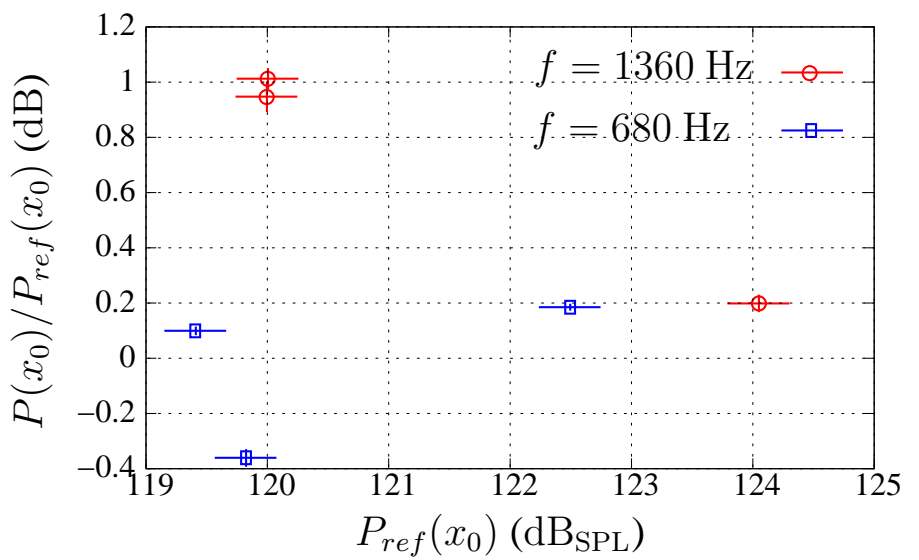

(b)

Figure 8. Amplitude of the ratio between the pressure estimated with LDV and the reference pressure as a function of the reference pressure for the different spacings $\Delta x$ shown in Table 2. Horizontal bars give information on the absolute uncertainty in the reference pressure and the vertical bars give information on the relative uncertainty in the pressure estimated by LDV. (a): acoustic level around $90 \mathrm{~dB}_{\mathrm{SPL}}$, (b): acoustic level around $120 \mathrm{~dB}_{\mathrm{SPL}}$. 
pressure) are in the interval $[-0.36 ; 1.07] \mathrm{dB}$ with an absolute minimum of 0.006 $\mathrm{dB}$.The largest biases could be due to large uncertainties in the estimated pressure but for both cases ( 92.7 and $120 \mathrm{~dB}$ ), the different results show a low variance. For this reason, we explain the largest biases by the errors made when calibrating the reference microphone at the end of the tube. Results show relative uncertainties in the reference pressure of around $0.2 \mathrm{~dB}$. The relative estimated pressure uncertainties are in the interval $[0.15 ; 1.27] \%$ corresponding to $[0.013 ; 0.11] \mathrm{dB}$ for the acoustic level between $[90 ; 124] \mathrm{dB}_{\mathrm{SPL}}$. Concerning the estimated phase, the uncertainties are in the interval $[0.34 ; 1.77]$ deg.

Results show a relatively good correlation between experimental uncertainties on the estimated pressure (amplitude, phase) and theoretical uncertainties obtained with the CRB. CRB give, for all the configurations tested, relative uncertainties on the amplitude of under $0.06 \mathrm{~dB}$ for an acoustic level greater than about $90 \mathrm{~dB}_{\mathrm{SPL}}$. For the phase, theoretical uncertainties are under $2.6 \mathrm{deg}$ for an acoustic level greater than $90 \mathrm{~dB}_{\mathrm{SPL}}$. Even if uncertainties obtained in the pressure estimated with LDV (amplitude $[0.013 ; 0.11] \mathrm{dB}$ and phase $[0.34 ; 1.77] \mathrm{deg}$ ) reach higher values for the amplitude than the theoretical uncertainties, the experimental and theoretical ranges are of the same order of magnitude.

\section{Conclusion}

This work deals with the estimation of acoustic pressure using acoustic velocity measured with LDV. A physical model of plane wave propagation in a waveguide is developed to calculate the pressure from two velocity measurements. This model is based on the mass conservation law. It can be seen as the equivalent of the model used for acoustic intensity measurement in the case of a 1D propagation model.

The theoretical minimum uncertainty in the LDV measured velocity given by the Cramer Rao Bounds is used in order to deduce the uncertainty in the pressure amplitude and phase. Moreover, this provides knowledge of the minimum measurable pressure level which is about $50 \mathrm{~dB}_{\mathrm{SPL}}$ for usual conditions encountered inside a waveguide (with small mean flow velocity).

The estimation technique is assessed experimentally using a tube excited by a loudspeaker. The uncertainty analysis is incorporated into the design of the experiment. To define the maximum admissible uncertainty, the optimal position for estimating the pressure, the optimal spacing between the two velocity measurements, the optimal frequency and the minimum acoustic level are determined.

The LDV estimated pressure is compared with reference pressure obtained from a microphone located at the end of the tube. Results show a bias in the interval $[-0.36 ; 1.07] \mathrm{dB}$ and a relative uncertainty in the pressure estimated by LDV of 
$[0.013 ; 0.11] \mathrm{dB}$. Bias and uncertainty values remain small (minimum bias of 0.006 $\mathrm{dB}$ and minimum relative uncertainty of $0.013 \mathrm{~dB}$ ) in some cases and show that it is possible to estimate the pressure with two velocity measurement in a waveguide excited with a plane wave. Large values of bias can be explained by errors that can occur when calibrating either the microphone or the LDV probe. Uncertainties observed experimentally show values broadly in agreement with the theoretical approach using the Cramer-Rao Bounds. For example, an acoustic level of 90 $\mathrm{dB}_{\mathrm{SPL}}$ leads to theoretical relative uncertainties under $0.06 \mathrm{~dB}$ and experimental relative uncertainties between $[0.03 ; 0.1] \mathrm{dB}$. As the acoustic level becomes higher, theoretical uncertainties in the pressure estimated by LDV diminish (level $>120$ $\mathrm{dB}_{\mathrm{SPL}}$, uncertainties $<0.015 \mathrm{~dB}$ ).

The absolute calibration of microphones can be performed with LDV measurements if the acoustic level is high enough, typically $120 \mathrm{~dB}_{\mathrm{SPL}}$. In these conditions, experimental uncertainties are in the interval $[0.013 ; 0.055] \mathrm{dB}$. This spread of values can be explained mainly by the fact that the two velocity measurements are not performed simultaneously and that the physical state of the system changes over the measurement duration. The uncertainty in the pressure estimation could therefore be lowered by using a system with two LDV probes measuring the two velocities at the same time.

\section{References}

[1] International Electrotechnical Commission IEC 61094-2:1992. Measurement microphones-Part2 : Primary method for the pressure calibration of laboratory standard microphones by the reciprocity method. International Electrotechnical Comission, 1992.

[2] C. Guianvarc'h, J-N. Durocher, M. Bruneau, and A-M. Bruneau. Improved Formulation of the Acoustic Transfer Admittance of Cylindrical Cavities. Acta Acustica, 21(3):345-499, 2006.

[3] C. Guianvarc'h, J-N. Durocher, M. Bruneau, and A-M. Bruneau. Acoustic transfer admittance of cylindrical cavities. J. Sound Vib., 292:595-603, 2006.

[4] S. Barrera Figueroa, K. Rasmussen, and F. Jacobsen. A time-selective technique for free-field reciprocity calibration of condenser microphones. J. Acoust. Soc. Am., 114(3):1467-1476, 2003.

[5] S. Barrera Figueroa, K. Rasmussen, and F. Jacobsen. On the interference between the two microphones in free-field reciprocity calibration. J. Acoust. Soc. Am., 116(5):2771-2778, 2004.

[6] International Electrotechnical Commission IEC 61094-3:1995. Measurement microphones-Part3 : Primary method for the free field calibration of laboratory standard microphones by the recsiprocity technique. International Electrotechnical Comission, 1995. 
[7] K.J. Taylor. Absolute measurement of acoustic particle velocity. J. Acoust. Soc. Am., 59(3):691-694, 1976.

[8] W. M. Humphreys, S. M. Bartram, T. L. Parrot, and M. G. Jones. Digital piv measurements of acoustic particle displacements in a normal incidence impedance tube. In 20th AIAA Advanced Measurement and Ground Testing Technology Conference, Albuquerque, June 15-18 1998.

[9] D.M. Campbell, J.A. Cosgrove, C.A. Greated, S.H. Jack, and D. Rockliff. Review of LDA and PIV applied to the measurement of sound and acoustic streaming. Optics \& laser technology, 32(8):629-639, 2000.

[10] K.J. Taylor. Absolute calibration of microphone by a laser-doppler technique. $J$. Acoust. Soc. Am., 70(4):939-945, 1981.

[11] T.J. MacGillivray, D.M. Campbell, C.A. Greated, and R. Barham. The Development of a Microphone Calibration Technique Using Laser Doppler Anemometry. AcusticaActa Acustica, 88(1):135-141, 2002.

[12] T.J. MacGillivray, D.M. Campbell, C.A. Greated, and R. Barham. The Development of a Microphone Calibration Technique Using Photon Correlation Spectroscopy. Acustica-Acta Acustica, 89(2):369-376, 2003.

[13] M.K. Mazumder, R.L. Overbey, and M.K. Testerman. Directional acoustic measurement by laser doppler velocimeters. Applied Physics Letters, 29(7):416-418, 1976.

[14] C.A Greated. Measurement of acoustic velocity fields. Strain, 22:21-24, 1986.

[15] B. Gazengel, 0. Richoux, and Ph. Rouquier. Characterization of a loudspeaker free field radiation by laser doppler velocimetry. Acta Acustica, 93(3):447-456, 2007.

[16] A. Degroot, R. MacDonald, J. Blondeau, M. Campbell, B. Gazengel, and O. Richoux. Etalonnage de microphones par Vélocimtrie Laser Doppler. In Congrès Francais d'Acoustique, Tours, France, 24-27 Avril 2006.

[17] A.M. Bruneau, M. Bruneau, P. Herzog, and J. Kergomard. Boundary layer attenuation of higher modes in waveguides. J. Sound Vib., 119(1):15-27, 1987.

[18] J.C. Pascal and C. Carles. Systematic measurement errors with two microphone sound intensity meters. J. Sound Vib., 83(1):53-65, 1982.

[19] B. Gazengel, S. Poggi, and J.C. Valière. Measurement of acoustic particle velocities in enclosed sound field: Assessment of two laser doppler velocimetry measuring systems. Applied Acoustics, 66(1):15-44, 2005.

[20] H. E. Albrecht, N. Damaschke, M. Borys, and C. Tropea. Laser Doppler and Phase Doppler Measurement Techniques. Springer Verlag, 2003.

[21] P. Flandrin. Time-Frequency, Time scale analysis. Academic press, 1998.

[22] B. Gazengel, S. Poggi, and J.C. Valière. Evaluation of the performance of two acquisition and signal processing systems for measuring acoustic particle velocities in air by means of laser doppler velocimetry. Meas. Sci. Technol., 14(12):2047-2064., 2003. 
[23] J.C. Valière, P. Herzog, V. Valeau, and G. Tournois. Acoustic velocity measurements in the air by means of laser Doppler velocimetry : dynamic and frequency range limitations and signal processing improvements. J. Sound and Vib., 229(3):607-626, 2000.

[24] V. Valeau, J.C Valière, and C. Mellet. Instantaneous frequency tracking of a sinusoidally frequency-modulated signal with low modulation index: application to laser measurements in acoustics. Signal Processing, 84(7):1147-1165, 2004.

[25] L. Simon, O. Richoux, A. Degroot, and L. Lionet. Laser doppler velocimetry joint measurements of acoustic and mean flow components : Lms-based algorithm. IEEE Transctions on Insrtumentation \& Measurements, 2007. accepted.

[26] A. Le Duff, G. Plantier, and J.C. Valière. A signal processing approach for acoustic velocity measurement. In Kando Conference, 2002. Budapest. 